\title{
Modifying the site index model of sugi planted forests in Miyazaki Prefecture considering the effects of DEM quality and scale of digital terrain analysis
}

\author{
Yasushi Mitsuda*1 and Satoshi Ito*1
}

\begin{abstract}
The objectives of this study were 1) to modify the site index prediction model for sugi (Cryptomeria japonica) planted forests in Miyazaki Prefecture developed in a previous study and 2) to investigate the effects of quality of DEM and the scale of digital terrain analysis on the performances of site index models. The study site was the Tano Forest Science Station, University of Miyazaki. We acquired 18 data sets of site index estimated using stem analysis and global position of each sample site where a sample tree was felled. Three topographic factors, a solar radiation index, a hydrological upslope contributing area index, and a vertical topographic exposure index, were derived from DEMs generated from three data sources $(10-\mathrm{m}$ and 50-m interval point data with 3D coordinates and digitized contour map) and at resolutions of 10, 12.5, 25, and $50 \mathrm{~m}$. Several search ranges (100, 250, 500, and $1000 \mathrm{~m}$ ) were tested. Correlation analysis between site index and topographic factors as well as regression analysis to develop a site index prediction model using topographic factors as explanatory variables revealed that the hydrological upslope contributing area index requires DEMs generated from more informative DEM data sources (10-m interval point data and digitized contour map) and at fine resolution (10 m or $12.5 \mathrm{~m}$ ); however, these DEMs were unsuitable for solar radiation index. DEMs generated from a less informative DEM data source (50 m interval 3D point data) and at a coarse resolution of $50 \mathrm{~m}$ were suitable for the solar radiation index. The effects of search ranges on topographic factors were clear for vertical topographic exposure index but not for the others. The best model developed in this study accepted the solar radiation index derived from the digital contour map based 10-m resolution DEM, the hydrological upslope contributing area index derived from the digital contour map based 12.5-m resolution DEM, and the vertical topographic exposure index derived from the $50-\mathrm{m}$ interval 3D point data based 50-m resolution DEM as the explanatory variables. The descriptive measures of model performance of this best model, namely $R^{2}$, adjusted $R^{2}$, and residual mean squared error, were $0.830,0.793$, and 1.522 , respectively. These values indicated that the best model of this study was superior to the previous one developed using only a digital contour map based 12.5-m resolution DEM.
\end{abstract}

keyword: digital elevation model, digital terrain analysis, scale problem, site index, sugi

\section{INTRODUCTION}

Predicting the spatial distribution of site productivity for a target planting species is essential for landscape-scale forest management (Kayahara et al., 1998; Klinka and Feller, 1984; Mitsuda et al., 2003; 2013; Takeshita et al., 1966). Digital terrain analysis (DTA) using a digital elevation model (DEM) can describe topographic features associated with plant

Corresponding author: Yasushi Mitsuda

${ }^{* 1}$ Faculty of Agriculture, University of Miyazaki, Miyazaki 889-2192, Japan

E-mail:mitsuda@cc.miyazaki-u.ac.jp (Y. Mitsuda) ecophysiological performance, which has been used to provide explanatory variables in modeling site productivity (e. g., Chen and Abe, 1999; Iverson et al., 1997; McNab, 1989; Minowa et al., 2005; Mitsuda et al., 2001; 2007; Munajati et al., 2009; Sturtevant and Seagle, 2004; Zushi, 2007). DTA is highly dependent on DEM quality, which affects the performance of site productivity prediction models. Using DEMs derived from various data sources and generated at various grid resolutions, we attempted to modify the site productivity model of sugi (Cryptomeria japonica) planted forests developed in our previous study (Mitsuda et al., 2007). We also investigated the effects of DTA scale on the site productivity model using this model modification procedure. These analyses revealed that site productivity models based on DTA were affected by DEM quality and DTA scale. Our findings will lead to a better understanding of the 
relationship of site productivity of sugi with topography; moreover, they will help in developing better site productivity models for other species and regions.

Topography is a key factor in representing plant responses to the environment and forest management (Takeshita et al., 1966). Site productivity, a concept used for representing a specific tree species' growth suitability for a given site (Davis and Johnson, 1987), has been studied with respect to topography. Site index, defined as the height of the dominant trees at a specific reference age (Davis and Johnson, 1987), is the most commonly used measure of site productivity for a target species (e.g., Davis and Johnson, 1987; Hägglund, 1981; Monserud et al., 1990; Takeshita et al., 1960; Wang, 1998). Various DTA-derived indices that represent topographic features, hereinafter referred to as topographic factors, have been used to develop site index prediction models (e.g., Iverson et al., 1997; Mitsuda et al., 2007; Zushi, 2007). Here we define three points to be considered in DTA: data source, grid resolution, and search range (Wilson et al., 2000). The term "search range" will be further explained in the following section. The value of a specific topographic factor for a specific site varies with respect to these three points; therefore, these points can affect site index model performance. DEM data sources are categorized into three data structures: regular grids, triangulated irregular networks, and line vectors representing contours. Such data sources are employed to generate a raster-type DEM that can be used in DTA. Several square-grid DEM data sources with various grid resolutions have been recently published, and some of them are usually available for a site of interest in Japan. However, a square-grid DEM source consists of summarized data that has lost detailed topographic information. Contour-type DEM data sources that do not lose topographic information can be used by digitizing the contour lines of the original topographic map; however, this is time-consuming and laborintensive. Thus, it is desirable to examine the effect of DEM data source on DTA in aspects of primary data structure (Van Niel et al., 2004; Wilson et al., 2000).

Scale remains an important but unsolved issue in scientific studies with spatial data, and scale problems defined by grid resolution and search range are important DTA-associated issues (Wilson et al., 2000). Determining a DEM's optimum grid cell size to represent topographic features with respect to target physical phenomena is a common scale problem (Hutchinson and Gallant, 2000; Sharma et al., 2011). Another scale problem is the determination of the optimum range for calculating topographic factors when the geomorphological pattern influences the target process of a site. Typically, algorithms used to calculate topographic factors with raster-type DEMs require an arbitrary value as a parameter (radius or window size) to define the range for searching a geomorphological feature that influences the physical phenomenon in the subject grid cell. Here we refer to the range (a circle or rectangle) defined by the given parameter as the search range.

In our previous study, we developed a site index model for sugi planted forests in Miyazaki Prefecture, Japan using
DTA-derived topographic factors (Mitsuda et al., 2007). Because DEM quality and the DTA scale problem were insufficiently considered in this previous study, we sought to modify the previous site index model while accounting for these two issues. The objectives of this study were 1) to evaluate the potential modification of the site index prediction model for sugi planted forests in Miyazaki Prefecture developed in a previous study and 2) to investigate the effects of quality of DEM and the scale of digital terrain analysis on the performances of site index models. Furthermore, we attempted to ascertain the appropriate DTA scale to represent each topographic feature with respect to site productivity.

\section{MATERIALS AND METHODS}

The study site was the Tano Forest Science Station, University of Miyazaki. The university forest (spread across $502 \mathrm{ha}$ ) is located in a lowland (approximately 110-300 $\mathrm{m}$ a.s.l.) of Miyazaki Prefecture, in a warm-temperate region in southeastern Kyushu Island, Japan, where the natural vegetation is evergreen broad leaved forest. The annual mean temperature and precipitation are $16.5^{\circ} \mathrm{C}$ and $2800 \mathrm{~mm}$, respectively. The site index data set used here is the same as that used in our previous study (Mitsuda et al., 2007). We felled the dominant tree in 18 sample sites where the stand age was greater than the reference age (40 years), and estimated the height of each dominant tree at the reference age as the site index by implementing Richards' threeparameter equation to the height-age data set derived from stem analysis. After felling, we recorded the position of each felled tree using DGPS (Trimble, Pathfinder ProXR). Thus, we acquired 18 data sets of precisely measured site index and global position of each sample site.

We used three DEM sources: 50-m and 10-m resolution square-grid DEMs published by the Japanese Geographic Survey Institute (GSI, now renamed the Geospatial Information Authority of Japan) and 10-m interval vectorized contour (hereafter, GSI50, GSI10, and CNT, respectively). Using 1:25000-scale topographic maps derived from manual stereoscopic interpretation of aerial photographs, GSI50 and GSI10 were obtained by reading the elevation on 50- and 10-m grids, and these two DEM data sources were treated as points with 3D coordinates at the center of grid cells. The CNT was obtained by digitizing the contour lines of the 1:25000-scale topographic maps of the study area, which maintained complete detailed topographic information of the 1:25000-scale topographic maps, whereas point-type data source (GSI50 and GSI10) lost it.

We interpolated the three data sources to yield rastertype DEMs with resolutions of $10,12.5,25$, and $50 \mathrm{~m}$ by the minimum curve method that fitted a two-dimensional cubic spline surface producing a smoothly varying surface. The four resolutions were set to compare our previous research (Mitsuda et al., 2007) using three of them $(12.5,25$, and $50 \mathrm{~m}$ resolution), and 10-m resolution square-grid DEM was newly published after the previous research. As various DEMs were used in this study, each DEM was identified by its data source and grid resolution. For example, GSI10-50 denoted a 
50-m resolution DEM derived from GSI10.

We focused on three environmental factors, namely solar radiation index (SRI), hydrological upslope contributing area index (UCA), and vertical topographic exposure index (VTEX) because these were selected as the explanatory variables of the best models in the regression analysis in our previous study. The SRI indicates the intensity of solar radiation that causes soil moisture deficiency, and it is proportional to the cosine of the solar incident angle (Smith et al., 1980; Mitsuda et al., 2001; 2007; Zushi, 2007). Solar incident angle is calculated by solar zenith, azimuth angle and land surface zenith, azimuth angle. The solar position with respect to the location of the sample site and time was estimated. The cosine of the solar incident angle was then calculated with respect to the solar position and land surface properties. The UCA reflects the tendency of water to accumulate in a catchment area (Beven and Kirkby, 1979; Mitsuda et al., 2001; 2007; Quinn et al., 1991; 1995). We determined the hydrological contributing area using a multiple-flow-direction algorithm to simulate soil water dynamics and weighted the UCA by slope angle and effective contour length to obtain the UCA used. This UCA was then converted into natural logarithm values and used in subsequent statistical analysis. VTEX is related to wind exposure and indicates the risk of forced evapotranspiration (e.g. McNab, 1993; Mitsuda et al., 2001; 2007; Quine and White, 1998; Takeshita et al., 1966; Yokoyama et al., 2002; Zushi, 2007). The depression angle to the lowest point was recorded for each of the eight main compass directions and then summed as VTEX, similar to Yokoyama's negative openness (e.g. Yokoyama et al., 2002).

We calculated the three topographic factors with search ranges of $100,250,500$, and $1000 \mathrm{~m}$. For calculating SRI, it was necessary to check whether the solar position at a given site and a given time was higher than the sky line; this was determined using surrounding elevation data within the assigned search range. For calculating UCA, the hydrological contributing area at a given site within the assigned search range was detected. For calculating VTEX, the lowest point for each direction for a given site within the assigned search range was found. As various topographic factors were used in this study, each topographic factor was identified by its index name, its assigned search range, and its DEM. For example, SRI-100_GSI10-50 denoted a SRI calculated with 100 -m search range using GSI10-50.

To detect trends in the correlation between the site index of sugi planted forests and each environmental factor with respect to various DEM sources, resolutions and search ranges, we calculated the correlation coefficients between the site index and the three environmental factors derived from each DEM defined by a combination of three sources and four resolutions.

We examined the effects of DEM quality and DTA scale on the performance of the site index model using a step-wise regression analysis. From the candidate models that comprised possible combinations of the three environmental factors, we selected the best linear regression model using Akaike's Information Criterion (AIC). First, we used the topographic factors derived from a DEM with the same source and resolution to construct candidate models. Next we used the topographic factors derived from DEMs with the same source but of various resolutions. Finally, we used the topographic factors derived from DEMs of all resolutions from all sources. In addition, we used the topographic factors derived from DEMs of all resolutions from GSI10 and GSI50, because of data availability.

\section{RESULTS}

Correlation coefficients between the site indices of sugi planted forests and environmental factors are shown in Fig. 1. In general, the site index was negatively correlated with SRIs and VTEXs. Hence, the Y-axes are inverted in the SRI and VTEX plots. The highest correlation coefficient (in absolute value) was 0.752 , observed for UCA-250_CNT-12.5. The correlation coefficients were higher for UCAs derived from DEMs generated from more informative DEM sources and in finer resolutions (CNT-10, CNT-12.5, GSI10-10 and GSI 10-12.5). They were lower for those derived from DEMs generated from CNT and GSI10 at coarse resolutions and from GSI50 at all resolutions. In contrast, the correlation coefficients between site index and SRI derived from GSI50 were better (lower) than those derived from CNT and GSI10. There were weaker correlations of site index with VTEXs than with UCAs and SRIs. The changes in correlation coefficients between site index and SRIs and UCAs with change in the search range were small, whereas the correlation coefficients between site index and VTEXs decreased significantly with increase in search range.

The descriptive measures of model performance (AIC, $R^{2}, R_{a}^{2}$, and RMSE) of the best linear regression models calculated for the best models of each DEM source and each grid resolution are shown in Table 1. The model performances improved with finer resolution for CNT and GSI10-based models, but worsened with finer resolution for GSI50-based models (Table 1). The accepted explanatory variables were SRI-100_CNT-10 and UCA-100_CNT-12.5 for the best model selected from alternative models of all possible combination of topographic factors derived from DEMs generated from CNT and in all resolutions, which we called here as the CNT best model, SRI-100_GSI10-12.5, UCA-250_GSI10, and VTEX-100_GSI10-10 for the GSI10 best model, and SRI-1000_GSI50-50 and VTEX-100_GSI50-50 for the GSI50 best model (Table 2). The best CNT model and the best GSI10 model adopted 10- or $12.5-\mathrm{m}$ resolution topographic factors, whereas the best GSI50 model adopted only 50-m resolution topographic factors. Among these three best models, the model performance was the finest for the best CNT model, followed by the best GSI10 model, and then the best GSI50 model. The best model among all possible candidates (the all-best model) and the best model among candidates derived from GSI10 and GSI50 (the best GSI model) adopted topographic factors derived from CNT-10, CNT-12.5, GSI-10, or GSI-50 (Table 3). Because UCA-250 GSI 10-10 were accepted in the best GSI10 model, we also calculated the descriptive measures of model performance of the second best GSI model using SRI-1000_GSI50-50, UCA250_GSI10-10, and VTEX-100_GSI50-50. The AIC of this model was 80.754 , indicating that model performance of the 

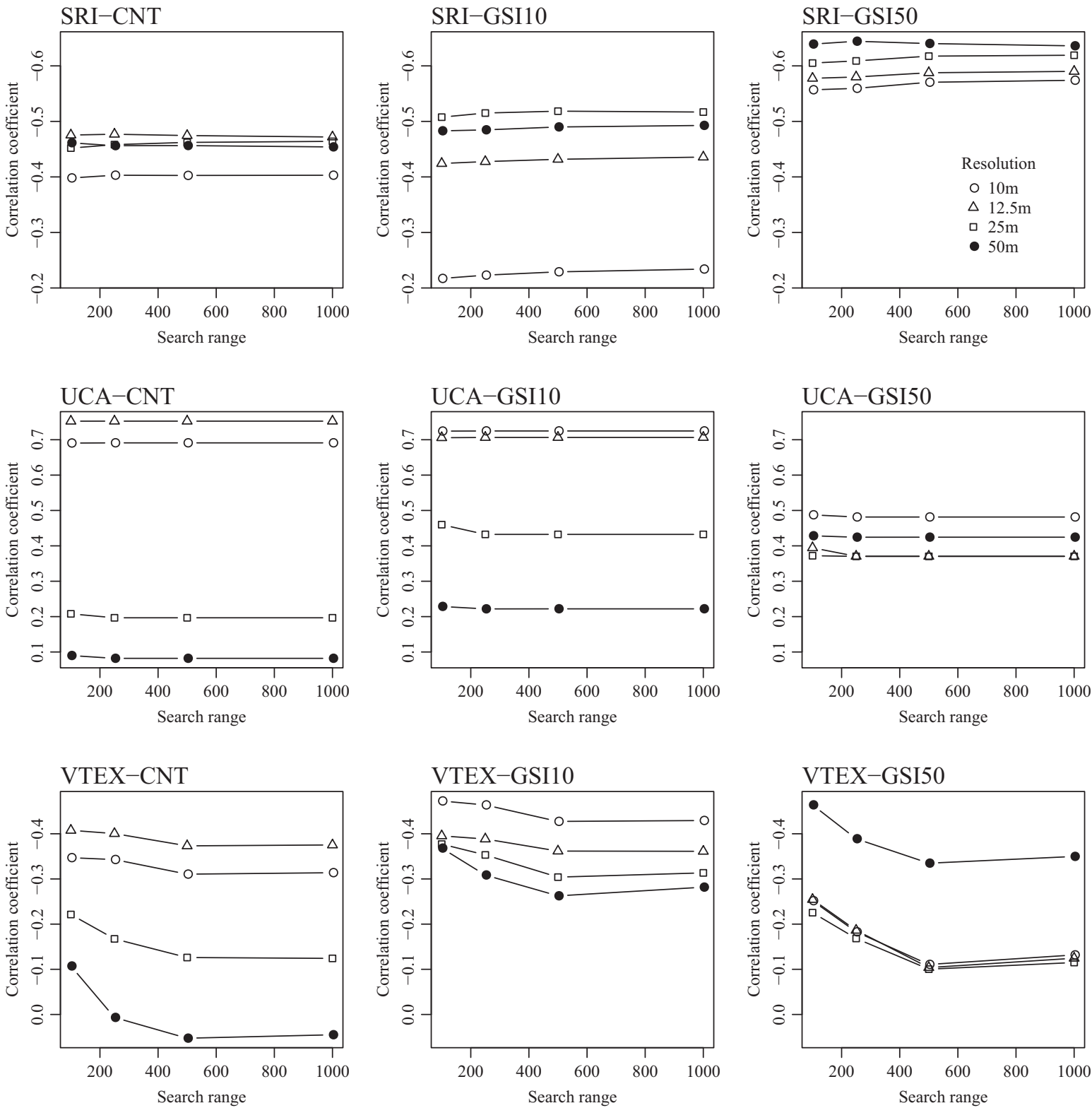

Fig. 1 Correlation coefficients between site index and topographic factors calculated using various search radii and data sources.

Table 1 Best site index model for each DEM source and resolution.

\begin{tabular}{ccccccccc}
\hline \multirow{2}{*}{ Source } & Resolution & SRI & $\begin{array}{c}\text { Variables } \\
\text { UCA }\end{array}$ & VTEX & AIC & $\mathrm{R}^{2}$ & $\mathrm{R}^{2}{ }^{2}$ & RMSE \\
\hline CNT & 10 & 100 & 250 & & 83.745 & 0.711 & 0.672 & 1.984 \\
& 12.5 & 100 & 100 & & 84.095 & 0.705 & 0.666 & 2.003 \\
& 25 & 1000 & & 100 & 96.339 & 0.418 & 0.340 & 2.815 \\
& 50 & 100 & & 100 & 96.160 & 0.424 & 0.347 & 2.800 \\
GSI10 & 10 & 1000 & 250 & & 88.559 & 0.622 & 0.572 & 2.268 \\
& 12.5 & 1000 & 100 & & 89.068 & 0.611 & 0.560 & 2.300 \\
& 25 & 1000 & & 100 & 96.437 & 0.415 & 0.337 & 2.823 \\
& 50 & 1000 & & 100 & 97.154 & 0.391 & 0.310 & 2.879 \\
GSI50 & 10 & 1000 & & 100 & 96.810 & 0.402 & 0.323 & 2.852 \\
& 12.5 & 1000 & & & 96.376 & 0.348 & 0.307 & 2.979 \\
& 25 & 1000 & & & 95.377 & 0.383 & 0.345 & 2.898 \\
& 50 & 1000 & & 100 & 86.637 & 0.660 & 0.615 & 2.145 \\
\hline
\end{tabular}


Table 2 Best site index model for each DEM source.

\begin{tabular}{cccccccc}
\hline \multirow{2}{*}{ Source } & \multicolumn{2}{c}{ Resolution, Search range } & \multirow{2}{*}{ AIC } & \multirow{2}{*}{$\mathrm{R}^{2}$} & \multirow{2}{*}{$\mathrm{R}^{2}{ }_{\mathrm{a}}$} & \multirow{2}{*}{ RMSE } \\
\hline CNT & SRI & UCA & VTEX & & & \\
GSI10 & 10,100 & $12.5,100$ & & 78.828 & 0.780 & 0.751 & 1.731 \\
GSI50 & 50,100 & 10,250 & 10,100 & 85.556 & 0.714 & 0.653 & 1.974 \\
\hline
\end{tabular}

Table 3 Best site index model among models using topographic factors derived from combinations of DEM sources and resolutions.

\begin{tabular}{cccccccc}
\hline & \multicolumn{2}{c}{ Source-Resolution, Search range } & \multirow{2}{*}{ AIC } & \multirow{2}{*}{ R $^{2}$} & \multirow{2}{*}{ R $^{2}{ }^{2}$} & \multirow{2}{*}{ RMSE } \\
\hline All & CNT $-10,1000$ & CNT-12.5, 100 & GSI50-50,100 & 76.199 & 0.830 & 0.793 & 1.522 \\
GSI & GSI50-50,1000 & GSI10-10,100 & GSI50-50,100 & 80.744 & 0.781 & 0.734 & 1.727 \\
\hline
\end{tabular}

second best GSI model was almost the same for the best GSI model (80.744). Thus, UCA-250_GSI10-10 can be considered as a good predictor variable for the site index model. Because we used the residual mean estimate (RME) in the previous study calculated as the mean absolute value of estimation error, we also calculated RME for the all-best model and the best GSI model. The values of $R^{2}, R^{2}{ }_{a}$, and RME were 0.692 , 0.651, and 1.618 for the previous model (Mitsuda et al., 2007), but those values were $0.830,0.793$, and 1.069 for the all-best model and $0.781,0.743$, and 1.376 for the best GSI model. Thus, the all-best model and the best GSI model were superior to the previous model.

\section{DISCUSSION}

The suitable DEM data source and grid resolution were different for each topographic factor with respect to its representation of a physical phenomenon (Beven and Kirkby, 1979; Hutchinson and Gallant, 2000; Quinn et al., 1995; Wilson et al., 2000). A high-quality DEM generated from more informative sources (CNT or GSI10) and at fine resolutions (10 m or $12.5 \mathrm{~m}$ ) was suitable for UCA, whereas a low-quality DEM generated from a less informative source (GSI50) and at coarse resolution $(50 \mathrm{~m})$ was suitable for SRI (Fig. 1). Topographic factors indicating microscale phenomena are more sensitive to DEM's capability to represent detailed topography than those indicating macroscale phenomena. Because UCA represents groundwater dynamic flow along a detailed topographical gradient, it can be regarded as a microscale topographic factor, and coarse-resolution DEMs are insufficient to describe groundwater flow influences by microscale topography. Because SRI represents solar radiation intensity determined by solar position, slope angle, and aspect, our results suggest that coarse-scale slope angle and aspect are suitable for evaluating SRI and then SRI can be regarded as a macroscale topographic factor. Coarse-scale slope angle and aspect that represent a macroscale trend in topography are suitable, however, fine-scale slope angel and aspect that are derived from high-quality DEMs are too detailed to represent intensity of solar radiation as a macroscale phenomenon. A fine-scale DEM will not be suitable for SRI because highly detailed topographical information will disturb to indicate this macroscale phenomenon. Thus, a suitable DEM grid resolution as a determinant factor of DTA scale is determined for a specific topographic factor.

Topographic characteristics of a target area will affect the importance of search range on DTA and development of a site index model. Correlation coefficients between site index and SRI and between site index and UCA saturated for search ranges, while those of VTEX worsened with longer search range in all cases (Fig. 1). The suitable search range for VTEX was obviously 100-m, irrespective of the DEM source and grid resolution. Because VTEX takes into account only the depression angle at the lowest point from each of the eight directions, it can be regarded as ridgeness, and thus, shorter search range was suitable for evaluating ridgeness in a highly undulated land area such as this study area. A longer search range may lead us to overestimate the ridgeness of a site because of a lower point in a further valley bottom beyond some ridges. The shorter search range will work well for SRI and UCA because of the highly undulating land surface of the study area. Although the effects of search range on DTA were not clear in the correlation analysis, our results suggest that search range will also affect DTA and site index modeling. The search ranges of UCA, SRI, and VTEX accepted in the best models developed in the stepwise approach were the same as $1000 \mathrm{~m}, 100$ or $250 \mathrm{~m}$, and $100 \mathrm{~m}$, respectively (Table 1,2, and 3). Thus, the DTA scale identified by the DEM grid resolution and search range affects the performance of a site index model.

A suitable DEM resolution corresponded to a specific DEM data source used in developing the site index model. As discussed above, UCA requires a fine-resolution DEM; however, the fine-resolution DEMs produced by upscaling from a low-quality source (such as GSI50-10 and GSI50-12.5) were not useful (Fig. 1 and Table 1). In contrast, SRI requires a coarse-resolution DEM, but the coarse-resolution DEMs produced by downscaling from high quality sources (such as CNT-50 and GSI10-50) were not useful (Fig. 1 and Table 1). In the best models developed for each DEM source, fineresolution topographic factors $(10 \mathrm{~m}$ or $12.5 \mathrm{~m}$ ) were accepted for the best CNT and GSI10 models, and coarse-resolution topographic factors $(50 \mathrm{~m})$ were accepted for the best GSI50 model. Our result suggests the need to choose an adequate grid resolution for DTA that corresponds to the quality of the original data source. In this case, CNT-10, CNT-12.5, GSI 10-10, and GSI50-50 are recommended. Furthermore, 
comparing correlation coefficients between site index and topographic factors derived from CNT-10 and CNT-12.5, 12.5 $\mathrm{m}$ resolution was more favorable than $10 \mathrm{~m}$. It may demonstrate the limit of resolution for topographic information contained in the 1:25000-scale topographic maps; moreover, $10 \mathrm{~m}$ resolution is too fine to represent topography on the basis of the 1:25000-scale topographic maps for this study area.

The results of stepwise regression analyses revealed that both DEM quality and DTA scale greatly affected the performance of the site index model of sugi planted forests. Although SRI-1000 CNT-10 was accepted in the all-best model, SRI-1000_GSI50, UCA-250_CNT12.5, UCA-250_GSI10, and VTEX-100_GSI50 were superior explanatory variables among UCAs, SRIs, and VTEXs in this study (Table 3). The descriptive measures of model performance (i. e., $R^{2}, R^{2}{ }_{a}$, and RME) indicated that the site index model of sugi planted forests developed in the present study was improved using the topographic factors derived from combinations of various DEM sources and various resolutions from the previous model, which used topographic factors produced by the DEM derived from a single data source and generated at fixed resolution. Our results show the importance of taking into account the DEM quality and DTA scale and testing various combinations of topographic factors derived from various DEM data sources, grid resolutions, and search ranges to develop site index models.

We developed a site index model of sugi planted forests for a relatively small area (approximately $500 \mathrm{ha}$ ) assuming that climatic condition was uniform within the target area. To develop a site index model for a broad area, we should take into account the climatic factors in the future studies (e.g., Klinka and Carter, 1990; Wang et al., 2004). Furthermore, because of geology is a factor that varies within a broad area and affects the physical and chemical characteristics of soil (Schoenholtz et al., 2000), it should be considered as well.

\section{CONCLUSION}

We improved the performance of the site index model of sugi planted forests in Miyazaki Prefecture by selecting explanatory variables from among topographic factors derived from DEMs generated from various sources and at various resolutions and calculated for various search ranges. Our results suggest that searching for suitable combinations of DEM source, resolution, and search range for specific topographic factors will contribute in developing better site index models. Our approach will also increase the awareness of the scales of topographic factors corresponding to each representing phenomenon such as soil water flow, solar radiation, wind flow, and so on, thereby leading to a better understanding of the autecology of target species.

\section{ACKNOWLEDGEMENTS}

This study was supported by JSPS KAKENHI Number 25850112, 25252029, and 15K07483. We would like to thank the two anonymous reviewers for their constructive comments.

\section{LITERATURE CITED}

Beven, K. J. and Kirkby, M. J. (1979) A physically based, variable contributing area model of basin hydrology. Hydrol. Sci. Bull. 24: 43-69

Chen, J. and Abe, N. (1999) Site classification for sugi plantation using GIS. J. For. Plann. 5: 1-8

Davis, L. S. and Johnson, K. N. (1987) Forest Management, McGraw-Hill, New York third edition

Hägglund, B. (1981) Evaluation of forest site productivity. For. Abst. 42: 515-527

Hutchinson, M. F. and Gallant, J. G. (2000) Digital elevation models and representation of terrain shape, In: Wilson, J. P. and Gallant, J. G. (eds) Terrain Analysis: principles and applications, John Wiley \& Sons, New York: 29-50

Iverson, L. R., Dale, M. E., Scott, C. T., and Prasad, A. (1997) A GIS-derived integrated moisture index to predict forest composition and productivity of Ohaio forests (U.S.A.). Landsc. Ecol. 12: 331-348

Kayahara, G. J., Klinka, K., and Marshall, P. L. (1998) Testing site index-site-factor relationship for predicting Pinus contorta and Picea engelmanii $\times P$. glauca productivity in central British Columbia. For. Ecol. Manage. 110: 141-150

Klinka, K. and Carter, R. E. (1990) Relationship between site index and synoptic environmental factors in immature coastal Douglas-fir stands. For. Sci. 36: 815-830

Klinka, K. and Feller, M. C. (1984) Principles used in selecting tree species for regeneration of forest sites in southwestern British Columbia. For. Chron. 60: 77-85

McNab, H. W. (1989) Terrain shape index: Quantifying effect of minor landforms on tree height. For. Sci. 35: 91-104

McNab, H. W. (1993) A topographic index to quantify the effect of mesoscale landform on site pruductivity. Can. J. For. Res. 23: 1100-1107

Minowa, Y., Suzuki, N., and Tanaka, K. (2005) Estimation of site indices with an artificial neural network. Jpn. J. For. Plann. 39: 23-38, (In Japanese with English abstract)

Mitsuda, Y., Ito, S., and Iehara, T. (2013) Methodology for regional forest reallocation using criteria and indicators of the Montreal Process. Landsc. Ecol. Manage. 18: 123-137, (In Japanese with English summary)

Mitsuda, Y., Ito, S., Matsushita, K., Fukuzato, K., Taniguchi, Y., Mizoue, N., and Shimizu, O. (2003) ESC (ecological site classification) based DSS for Japabese forest landscape suffering frequent rainstorms, In: CD Proceedings of a Transdisciplinary Conference on Decision Support for Multiple Purpose Forestry, Vienna

Mitsuda, Y., Ito, S., and Sakamoto, S. (2007) Predicting the site index of sugi plantations from GIS-derived environmental factors in Miyazaki Prefecture. J. For. Res. 12: 177-186

Mitsuda, Y., Yoshida, S., and Imada, M. (2001) Use of GISderived environmental factors in predicting site indices in Japanese larch plantations in Hokkaido. J. For. Res. 6: 87-93

Monserud, R. A., Moody, U., and Breuer, D. W. (1990) A soilsite study for inland Douglas-fir. Can. J. For. Res. 20: 686-695 Munajati, S. L., Abe, N., and Tsukahara, M. (2009) Predicting Site Indices of Japanese Cedar Plantation in Niigata Prefecture using Environmental Factors. J. For. Plann. 15: 
$45-51$

Quine, C.P. and White, I. M.S. (1998) The potential of distancelimited topex in the prediction of site windiness. Forestry 71: 325-332

Quinn, P., Beven, K., Chevallier, P., and Planchon, O. (1991) The prediction pf hillslope flow path for distributed hydrological modelling using digital terrain models. Hydrol. Process. 5: 59-79

Quinn, P., Beven, K., and Lamb, R. (1995) The $\ln a / \tan \beta$ index: How to calculate it and how to use it within the topmodel framework. Hydrol. Process. 9: 161-182

Schoenholtz, S. H., Miegroet, H. V., and Burger, J. A. (2000) A review of chemical and physical properties as indicators of forest soil quality: challenges and opportunities. For. Ecol. Manage. 138: 335-356

Sharma, A., Tiwari, K. N., and Bhadoria, P. B. S. (2011) Determining the optimum cell size of digital elevation model for hydrologic application. J. Earth. Syst. Sci. 120: 573-582

Smith, J. A., Lin, T. L., and Ranson, K. J. (1980) The lambertian assumption and landsat data. PE \& RS 46: 1183-1189

Sturtevant, B. R. and Seagle, S. W. (2004) Comparing estimate of forest site quality in old second-growth oak forests. For. Ecol. Manage. 19: 311-328

Takeshita, K., Fukushima, T., Hagiwara, Y., and Saijo, T. (1966) Stand analysis concerning estimation of forest-land productivity. Bull. Fukuoka-pref For. Exp. Stn. 18: 41-76, (In Japanese with English summary)

Takeshita, K., Nakanjima, Y., Nagahama, M., and Higuchi, S.
(1960) Edaphological studies on micro-topography and forest soil and their relationship to the growth of sugi Cryptmeria races (I). Bull. Fukuoka-pref. For. Exp. Stn. 12: 1-162, (In Japanese with English summary)

Van Niel, K. P., Laffan, S. W., and Lees, B. G. (2004) Effect of error in the DEM on environmental variables for predictive vegetation modelling. J. Veg. Sci. 15: 747-756

Wang, G. G. (1998) Is height of dominant trees at a reference diameter an adequate measure of site quality? For. Ecol. Manage. 112: 49-54

Wang, G. G., Huang, S., Monserud, R. A., and Klos, R. J. (2004) Lodgepole pine site index in relation to synoptic measures of climate, soil moisture and soil nutrients. For. Chron. 80: 678-686

Wilson, J. P., Repetto, P. L., and Snyder, R. D. (2000) Effect of data source, grid resolution, and flow-routing method on computed topographic attributes, In: Wilson, J. P. and Gallant, J. G. (eds) Terrain Analysis: principles and applications, John Wiley \& Sons, New York: 133-161

Yokoyama, R., Shirasawa, M., and Pike, R. J. (2002) Visualizing topography by openness: A new application of image processing to digital elevation models. PE \& RS 68: 257-265

Zushi, K. (2007) Regional estimation of Japanese cedar (Cryptomeria japonica d. don) productivity by use of digital terrain analysis. J. For. Res. 12: 289-297

(Received 1 August 2015)

(Accepted 29 October 2015) 